\title{
Complémentation d'une paille de blé avec des blocs multinutritionnels : effets sur la digestibilité de la paille et intérêt pour des brebis taries et des agneaux en croissance
}

\author{
Mohamed Houmania*, Jean-Louis Tisserand ${ }^{\mathrm{b}}$
}

\author{
a Institut d'agronomie, Université de Blida, BP 270, Route de Souma, 09100 Blida, Algérie \\ ${ }^{\mathrm{b}}$ École nationale d'enseignement supérieur d'agronomie, BP 1607, 26036 Dijon cedex, France
}

(Reçu le 12 février 1998 ; accepté le 14 septembre 1998)

\begin{abstract}
Supplementation of wheat straw with multinutritional blocks: effects on the digestibility of straw and interest for dry ewes and growing lambs. Two blocks characterized by the presence of olive cake in the first block (BMN1) and dropping of poultry in the second block (BMN2) were compared with a classical concentrate (C). The BMN1 or BMN2 blocks were characterized by a high mincral matter (26-31\%) and crude protein (2l-22\%) level. In vitro digestibility of the two blocks were different, advantaging the BMN2 block. These blocks, fed with straw (P) to adult sheep, dry ewes and growing lambs, improved straw ingestibility in similar quantities compared to the concentrate. Straw supplemented with the BMN1 block or BMN2 block increased the ad libitum intake by $21 \%$ in contrast to straw without supplement. Straw in vivo digestibility was increased by the supplementation with the blocks. Ration nitrogen balance (P+BMN2) with $30.0 \%$ was higher compared to ration $(\mathrm{P}+\mathrm{BMN} 1)$ with $27.0 \%$ and similar to the ration $(\mathrm{P}+\mathrm{C})$. The $(\mathrm{P}+\mathrm{BMN} 2)$ ration allowed better results with dry ewes ( $5.9 \mathrm{~g}$ per day) and with growing lambs ( $105 \mathrm{~g}$ per day). The $(\mathrm{P}+\mathrm{BMN} 1)$ ration allowed one weight gain to $83 \mathrm{~g}$ per day and reduced the live weight of $8.4 \mathrm{~g}$ per day with dry ewes. The ration containing the BMN1 block and BMN2 block enabled a saving of, respectively, 13.6 and $16.7 \%$ on the feeding cost per day with dry ewes and 12 and $23 \%$ on the feeding cost per $\mathrm{kg}$ of weight gain with growing lambs compared to the ration containing the concentrate. (@) Elsevier/Inra)
\end{abstract}

straw / multinutritional blocks / feeding value / nitrogen balance / ewes / growing lambs

Résumé - Deux types de blocs contenant respectivement des grignons bruts d'olives (BMN1) et des fientes de volailles (BMN2) ont été comparés à un aliment concentré classique. Les digestibilités in vitro des blocs BMN1 ont été inférieures à celles des blocs BMN2, elles-mêmes inférieures à celles du concentré (C). Après complémentation avec les blocs (BMN1 et BMN2) et le concentré (C), les quantités de paille ingérées par les moutons ont augmenté respectivement de 17 et $21 \%$. Les diges-

\footnotetext{
* Correspondance et tirćs à part
} 
tibilités in vivo (dMO, dCB et dMAT) de la paille (P) complémentée avec les blocs ont été peu différentes de celles observées avec la complémentation classique (C). Les rétentions azotées avec les rations $(\mathrm{P}+\mathrm{C})$ et $(\mathrm{P}+\mathrm{BMN} 2)$ ont été identiques et plus élevées qu'avec la ration $(\mathrm{P}+\mathrm{BMN} 1)$. La ration ( $\mathrm{P}+\mathrm{BMN} 2)$ a permis d'entretenir correctement des brebis taries de $48-49 \mathrm{~kg}$ de poids vif alors qu'avec les rations $(\mathrm{P}+\mathrm{C})$ et $(\mathrm{P}+\mathrm{BMN} 1)$, les brebis ont perdu un peu de poids vif. Le gain de poids vif le plus élevé ( $105 \mathrm{~g} \cdot \mathrm{j}^{-1}$ ) a été observé chez les agneaux ayant reçu la ration ( $\mathrm{P}+\mathrm{BMN} 2$ ) et le plus faible $\left(83,6 \mathrm{~g} \cdot \mathrm{j}^{-1}\right)$ chez ceux ayant reçu la ration $(\mathrm{P}+\mathrm{BMN} 1)$. Les rations renfermant les blocs $(\mathrm{P}+\mathrm{BMN} 1)$ et $(\mathrm{P}+\mathrm{BMN} 2)$ comparées aux rations $(\mathrm{P}+\mathrm{C})$, ont permis des économies de 13,6 et $16,7 \%$ sur le coût alimentaire des brebis taries et de 12 et $23 \%$ sur celui du $\mathrm{kg}$ de gain de poids vif des agneaux. (@ Elsevier / Inra)

paille / blocs multinutritionnels / valeur alimentaire / bilan azoté / brebis / agneaux / croissance

\section{INTRODUCTION}

Dans les pays du sud de la Méditerranée, les résidus lignocellulosiques, particulièrement les pailles de céréales, représentent une source importante d'aliment pour les ruminants. En Algérie, la demande est considérable durant la longue période sèche (mai à octobre), période pendant laquelle la paille de céréales peut constituer l'essentiel de la ration de base des ruminants. Selon Sansoucy et al. [17], Hassoun [8] et Nyarko-Badohu et al. [15], la valeur alimentaire des pailles peut être améliorée par une complémentation constituée de sous-produits associés en blocs multinutritionnels dont la technique de fabrication est relativement simple. Encore peu développée, l'industrie agro-alimentaire algérienne, dégage déjà annuellement quelque $1300000 \mathrm{t}$ de fientes de volailles, $75000 \mathrm{t}$ de grignons bruts d'olives et $195000 \mathrm{t}$ de sons de céréales. Il s'y ajoute 17000 t de mélasse de canne à sucre d'importation. À l'exception des sons, les autres sous-produits ne trouvent pas de débouchés réels. Les grignons bruts d'olives et les fientes de volailles auxquelles on peut ajouter la litière de poulets de chair sont simplement jetés ou brûlés. C'est la raison pour laquelle nous avons entrepris l'étude de l'incorporation des fientes de volailles et des grignons d'olives dans des blocs multinutritionnels renfermant également de la mélasse de canne et du gros son de blé pour la complémentation de la paille de blé dur.
Dans cette étude, sont rapportés les résultats de trois essais. Dans 1'essai 1, la composition chimique et la digestibilité in vitro des blocs multinutritionnels ont été déterminés. Dans l'essai 2, l'effet des blocs sur la digestibilité in vivo de la paille a été mesuré. Dans l'essai 3, des régimes constitués de blocs en complémentation d'une paille de blé pour l'alimentation de brebis taries et d'agneaux en croissance ont été testés.

\section{MATÉRIEL ET MÉTHODES}

\subsection{Paille}

Il s'agit d'une paille de blé (Triticum durum des : variété locale Oued Zenati) récoltée dans la localité de Berrouaghia à $80 \mathrm{~km}$ au sud d'Alger.

\subsection{Essai 1 : Composition chimique et digestibilité in vitro des blocs multinutritionnels et du concentré}

Les blocs ont été fabriqués selon deux formules : bloc multinutritionnel 1 (BMN1) renfermant des grignons bruts d'olives et bloc multinutritionnel 2 (BMN2) renfermant des fientes de volaille. La technique de fabrication utilisée est celle rapportée par Hassoun [8]. Un aliment concentré (C) dont la composition centésimale est comparable à celle utilisée en Algérie, a été également étudić (tableau I). Les digestibilités in vitro de la matière organique (DivMO) et de la 
Tableau I. Composition centésimale des blocs multinutritionnels et du concentré.

\begin{tabular}{lccc}
\hline Aliments & Concentré (C) & Bloc (BMN1) & Bloc (BMN2) \\
\hline Fientes & - & - & 17,82 \\
Grignons & - & 16,85 & - \\
Mélasse & - & 35,17 & 35,17 \\
Orge & 61,25 & - & - \\
Gros son & 25,84 & 25,84 & 25,84 \\
Farine de viande & 8,52 & 3,39 & 3,39 \\
Soufre & 0,23 & 0,23 & 0,23 \\
Urée & 1,18 & 4,46 & 3,49 \\
Ciment & - & 11,04 & 11,04 \\
CMV & 1,00 & 1,04 & 1,04 \\
Sel & 0,83 & 0,83 & 0,83 \\
Calcaire & 1,15 & 1,15 & 1,15 \\
\hline
\end{tabular}

$\mathrm{C}=$ concentré classique $;$ blocs $\mathrm{BMN} 1$ et $\mathrm{BMN} 2=$ blocs multinutritionnels 1 et $2 ; \mathrm{CMV}=$ complément minéral vitaminé.

cellulose brute (DivCB) des blocs et du concentré ont été déterminées par la technique de Tilley et Terry [19] suivant le schéma rapporté par Nefzaoui et Vanbelle [14]. Trois moutons de race locale « Ouled Djellal » porteurs d'une canule ruminale, ont servi d'animaux donneurs de jus de rumen. Ils ont été alimentés avec la paille de blé dur complémentée à chaque fois avec le complément étudié (320 g.j $\mathrm{j}^{-1}$ de C ; $277 \mathrm{~g} \cdot \mathrm{j}^{-1} \mathrm{de}$ BMN1 et $299 \mathrm{~g} \cdot \mathrm{j}^{-1}$ de BMN2). La paille a été offerte à volonté ( 10 à $15 \%$ de refus). Le jus de rumen a été prélevé $3 \mathrm{~h}$ après la distribution du repas du matin. Les digestibilités des MAT ont été calculées à partir des tables des aliments de l'Inra [9].

\subsection{Essai 2 : Effet des blocs sur la digestibilité in vivo d'une paille de blé dur}

La digestibilité in vivo a été mesurée sur cinq béliers adultes non castrés, de race locale «Ouled Djcllal », pesant en moyenne $58 \mathrm{~kg}$, placés dans des cages à métabolisme, au cours de quatre périodes successives de $21 \mathrm{j}$ séparées par une période de transition de $7 \mathrm{j}$. Il s'agit de la même méthodologie de mesure que celle pratiquée à I'Inra de Theix [3]. Les animaux ont reçu successivement la paille non complémentée $(P)$, la paille + concentré $(\mathrm{P}+\mathrm{C})$, la paille + bloc 1 $(\mathrm{P}+\mathrm{BMN} 1)$ et la paille + bloc $2(\mathrm{P}+\mathrm{BMN} 2)$. $\mathrm{La}$ paille a été distribuée à volonté $(10$ à $15 \%$ de refus) en deux repas par jour ( 10 et $16 \mathrm{~h}$ ). Le concentré a été offert à raison de $320 \mathrm{~g} \cdot \mathrm{j}^{-1}$ en deux repas $(9$ et $14 \mathrm{~h}$ ). Les blocs ont été mis à la disposition des animaux 20 min le matin ( 9 à $9 \mathrm{~h} 20 \mathrm{mn}$ ) et 20 min l'après-midi ( 14 à 14 h 20 $\mathrm{mn}$ ) de façon à leur permettre un niveau d'alimentation proche de l'entretien, soit $277 \mathrm{~g} \cdot \mathrm{j}^{-1} \mathrm{de}$ bloc BMN1 et $299 \mathrm{~g} \cdot \mathrm{j}^{-1}$ de bloc BMN2. Les animaux ont disposé d'eau à volonté. La digestibilité de la paille a été calculée par différence à partir de celle de la ration entière en utilisant les valeurs de la digestibilité in vitro des blocs et du concentré obtenues dans l'essai 1. L'azote retenu a été déterminé pour les rations.

\subsection{Essai 3 : Effet des régimes paille + blocs sur les performances des brebis taries et des agneaux en croissance}

Trente brebis taries de race locale « Ouled Djellal » âgées d'environ 3 ans ont été choisies en fonction de leur poids vif dans un troupeau de plus de 100 têtes. Elles ont été identifiées et réparties au hasard en trois lots de dix animaux chacun et de poids vifs moyens comparables $(48,6 \pm$ $0,5 \mathrm{~kg}$ ). Trente agneaux de la même race ont été choisis en fonction de leur âge (environ $105 \mathrm{j}$ ) et de leur poids vif dans un troupeau de plus de 120 têtes. Ils ont été identifiés et répartis au hasard en trois lots de dix animaux chacun et de 
poids vifs moyens comparables $(22,0 \pm 0,4 \mathrm{~kg})$ Les brebis et les agneaux retenus pour l'essai ont été traités contre les parasites internes et externes.

Pour les brebis et les agneaux, le lot 1 a reçu de la paille complémentée avec le concentré (C) et les lots 2 et 3 ont reçu de la paille complémentée respectivement avec le bloc BMN1 et le bloc BMN2. Pour les brebis, la paille a été offerte à volonté en deux repas par jour (10 et $16 \mathrm{~h}$ ); le concentré $(\mathrm{C})$ a été distribué en deux repas par jour $\left(9\right.$ et $14 \mathrm{~h}$ ) à raison de $219 \mathrm{~g} \cdot \mathrm{j}^{-1} \cdot$ repas $^{-1}$; les blocs ont été mis à la disposition des animaux 5 à $6 \mathrm{~h}$ par jour. Pour les agneaux, la paille a été distribuée à volonté en deux repas par jour (10 et $16 \mathrm{~h}$ ); le concentré en deux repas par jour (9 et $14 \mathrm{~h}$ ) alors que les blocs ont été en permanence à la disposition des animaux.

Les animaux ont été pesés individuellement à jeun tous les $20 \mathrm{j}$. Les aliments distribués et les refus ont été pesés chaque jour. Les blocs ont été pesés à leur mise à la disposition des animaux.

La durée de l'essai a été de $121 \mathrm{j}$ dont $21 \mathrm{j}$ d'adaptation des animaux aux régimes alimentaires.

\subsection{Analyses chimiques}

Les teneurs en matière sèche (MS), cendres $(\mathrm{MM})$, cellulose brute (CB) et matières azotées totales (MAT) ont été déterminées selon les méthodes de l'AOAC [1]. Toutes les mesures ont été effectuées en triple.

\subsection{Analyses statistiques}

Une analyse de variance a été effectuée par le test de Fischer et une comparaison des moyennes par le test de Newman-Keuls.

\section{RÉSULTATS}

\subsection{Composition chimique des sous-produits et des blocs multinutritionnels}

Les grignons d'olives ont une teneur en CB élevée $(48,7 \%)$, supérieure de 9,6 points à celle de la paille, une teneur en MAT $(7,8 \%)$ double de celle de la paille et une teneur en MM $(2,2 \%)$ relativement faible.
Les fientes de volailles ont une teneur élevée en MAT, près de quatre fois plus élevée que celle des grignons, une teneur en MM près de huit fois plus élevée que celle des grignons et une teneur en $\mathrm{CB}$ près de trois fois inférieure à celle des grignons (tableau $I$ ).

Les blocs multinutritionnels (BMN1 et BMN2) ont une teneur en MS inférieure à celle du concentré $(C)$ et des teneurs plus élevées en MAT et en MM. La teneur en $\mathrm{CB}$ du concentré est comprise entre celles des blocs BMN1 et BMN2 (tableau II).

\subsection{Essai 1 : Digestibilité in vitro des blocs multinutritionnels et du concentré}

Les blocs BMN2 sont plus digestibles que les bloes BMN1 : $+5,6$ points pour la DivMO et $+22,2$ points pour la DivCB. La DivCB du concentré $(C)$ est supérieure de 5,6 points et inférieure de 16,6 points à celle respectivement des blocs $\mathrm{BMN} 1$ et $\mathrm{BMN} 2$. $\mathrm{La}$ DivMO du concentré $(C)$ est supérieure à celle des deux blocs. La digestibilité des MAT (calculée à partir des tables Inra, 1988) des blocs BMN2 est plus élevée de 10,5 points que celle des blocs BMN1 et inférieure de 5,6 points à celle du concentré (C) (tableau III).

\subsection{Essai 2 : Effet des blocs sur la digestibilité in vivo d'une paille de blé dur}

\subsubsection{Ingestibilité de la paille, des blocs multinutritionnels et du concentré}

Pour la paille distribuée seule, la quantité ingérée est de $31,1 \mathrm{~g} \mathrm{MS} \cdot \mathrm{kg}^{-1} \mathrm{P}^{0.75}$ (tableau $I V)$; sa complémentation a entraîné une augmentation significative de son ingestion de 20,5 à $21,5 \%$ avec les blocs (BMN1 et BMN2) et de $16,7 \%$ avec le concentré (C). Les niveaux d'ingestion de la paille complémentée avec les trois compléments ne sont pas significativement différents entre eux. 
Tableau II. Composition chimique des blocs multinutritionnels, du concentré, de leurs constituants et de la paille.

\begin{tabular}{lcrrrr}
\hline & & \multicolumn{5}{c}{ Teneurs en \% de MS } \\
\cline { 3 - 6 } Aliments & MS $(\%)$ & MO & MAT & CB & MM \\
\hline Paille & 91,1 & 94,0 & 3,3 & 39,1 & 6,0 \\
C & 90,0 & 89,3 & 17,8 & 7,0 & 10,7 \\
BMN1 & 84,2 & 73,3 & 22,2 & 10,6 & 26,7 \\
BMN2 & 84,2 & 69,4 & 21,5 & 4,9 & 30,6 \\
Orge & 89,0 & 95,8 & 11,8 & 7,5 & 4,2 \\
Fientes' & 90,0 & 82,0 & 29,4 & 17,0 & 18,0 \\
Grignons bruts & 90,0 & 97,8 & 7,8 & 46,7 & 2,2 \\
Mélasse & 75,3 & 92,6 & 5,5 & - & 7,3 \\
Gros son & 87,0 & 94,0 & 17,0 & 11,1 & 6,0 \\
Farine de viande & 96,0 & 71,3 & 35,0 & - & 28,7 \\
CMV & 92,2 & & & & \\
Calcaire & 91,6 & & & & \\
Sel & 89,7 & & & & \\
Soufre & 95,9 & & & & \\
Urée & 92,0 & & & & \\
Ciment & 97,0 & & & & \\
\end{tabular}

$\mathrm{MS}=$ matière sèche $; \mathrm{MO}=$ matière organique $; \mathrm{MAT}=$ matières azotées totales $; \mathrm{CB}=$ cellulose brute $;$ $\mathrm{MM}=$ matières minérales $; \mathrm{CMV}=$ complément minéral vitaminé $; \mathrm{C}=$ concentré classique ; $\mathrm{BMN} 1$ et BMN2 = blocs multinutritionnels 1 et $2 ;{ }^{1}=$ sous-produits déshydratés.

Tableau III. Digestibilité in vitro des blocs multinutritionnels et du concentré.

\begin{tabular}{|c|c|c|c|}
\hline \multirow{2}{*}{$\begin{array}{l}\text { Digestibilité } \\
(\%)\end{array}$} & \multicolumn{2}{|c|}{ Digestibilité in vitro } & \multirow{2}{*}{$\begin{array}{c}\text { Digestibilité calculée } \\
\text { dMAT }\end{array}$} \\
\hline & DivMO & DivCB & \\
\hline BMN I & $66,4 \pm 1,6^{\mathrm{a}}$ & $27,6 \pm 1,2^{a}$ & $53,1 \pm 3,7^{a}$ \\
\hline BMN2 & $72,2 \pm 2,5^{b}$ & $49,8 \pm 2,3^{b}$ & $63,6 \pm 2,0^{\mathrm{h}}$ \\
\hline $\mathrm{C}$ & $77,4 \pm 3,4^{c}$ & $33,2 \pm 1,3^{\mathrm{c}}$ & $69,2 \pm 3,6^{c}$ \\
\hline
\end{tabular}

$\mathrm{C}=$ concentré classique $; \mathrm{BMN} 1$ et $\mathrm{BMN} 2=\mathrm{Blocs}$ multinutritionnels 1 et $2 ; \mathrm{MO}=$ matière organique ; MAT = matières azotées totales $; \mathrm{CB}=$ cellulose brute $;$ divMO = digestibilité in vitro de la $\mathrm{MO} ;$ divCB $=$ digestibilité in vitro de la $\mathrm{CB} ; \mathrm{dMAT}=$ digestibilité des MAT ${ }^{\prime}{ }^{\prime}=$ valeurs calculées à partir des tables Inra (1988); sur une même colonne, les valeurs marquées d'un même indice sont comparables au seuil de $5 \%$.

Les blocs multinutritionnels sont d'autant plus ingérés qu'ils sont plus longtemps mis à la disposition des animaux. Ils ont été ingérés entre 11,1 et $12,0 \mathrm{~g} \mathrm{MS} \cdot \mathrm{kg}^{-1} \mathrm{P}^{0.75}$ par les moutons en $40 \mathrm{~min}$, entre 36,2 et
$37,3 \mathrm{MS} \cdot \mathrm{kg}^{-1} \mathrm{P}^{0.75}$ par les brebis en 5 à $6 \mathrm{~h}$ et entre 61,9 et $64,5 \mathrm{~g} \mathrm{MS} \cdot \mathrm{kg}^{-1} \mathrm{P}^{0.75}$ par les agneaux qui en disposaient de façon permanente. Le concentré (C), offert en quantité limitée, a été ingéré en quantités inférieures à 
Tableau IV. Ingestibilité de la paille, des blocs et du concentré et digestibilité in vivo de la paille chez les moutons.

\begin{tabular}{lcccc}
\hline Régimes & $\mathrm{P}$ & $\mathrm{P}+\mathrm{C}$ & $\mathrm{P}+\mathrm{BMN1}$ & $\mathrm{P}+\mathrm{BMN} 2$ \\
\hline Ingestibilité $\left(\mathrm{gMS} \cdot \mathrm{kg}^{-1} \mathrm{P}^{0,75}\right)$ & & & & \\
$\quad$ Ration entière & & $50,1 \pm 1,9^{\mathrm{a}}$ & $48,6 \pm 1,5^{\mathrm{b}}$ & $49,7 \pm 1,5^{\mathrm{a}}$ \\
$\quad$ Paille seule & $31,1 \pm 1,6^{\mathrm{b}}$ & $36,3 \pm 1,6^{\mathrm{a}}$ & $37,5 \pm 1,7^{\mathrm{a}}$ & $37,7 \pm 1,7^{\mathrm{a}}$ \\
Bloc ou concentré & & $13,8 \pm 1,4^{\mathrm{a}}$ & $11,1 \pm 1,2^{\mathrm{b}}$ & $12,0 \pm 1,2^{\mathrm{c}}$ \\
& & & & \\
Digestibilité (\%) & & & \\
$\quad$ Ration entière & & & \\
$\quad$ - dMO & $44,4 \pm 1,2^{\mathrm{c}}$ & $50,6 \pm 0,9^{\mathrm{a}}$ & $56,7 \pm 1,4^{\mathrm{b}}$ & $59,1 \pm 1,9^{\mathrm{b}}$ \\
- dMAT & $9,9 \pm 1,3^{\mathrm{c}}$ & $59,5 \pm 2,3^{\mathrm{b}}$ & $54,0 \pm 2,8^{\mathrm{a}}$ & $60,9 \pm 3,1^{\mathrm{b}}$ \\
Paille seule & & & & \\
- dMO & & & \\
- dMAT & $44,4 \pm 1,2^{\mathrm{d}}$ & $48,9 \pm 1,3^{\mathrm{a}}$ & $49,8 \pm 1,7^{\mathrm{a}}$ & $49,8 \pm 1,5^{\mathrm{a}}$ \\
- dCB & $9,9 \pm 1,3^{\mathrm{d}}$ & $18,8 \pm 1,9^{\mathrm{a}}$ & $17,4 \pm 1,4^{\mathrm{b}}$ & $17,9 \pm 1,3^{\mathrm{b}}$ \\
& $53,3 \pm 1,7^{\mathrm{d}}$ & $57,3 \pm 0,4^{\mathrm{b}}$ & $56,4 \pm 0,4^{\mathrm{b}}$ & $58,9 \pm 0,3^{\mathrm{a}}$ \\
\hline
\end{tabular}

$\mathrm{P}=$ paille $; \mathrm{C}=$ concentré classique $; \mathrm{BMN} 1$ et $\mathrm{BMN} 2=$ blocs multinutritionnels 1 et $2 ; \mathrm{dMO}=$ digestibilité in vivo de la matière organique; $\mathrm{dMAT}=$ digestibilité in vivo des matières azotées totales; $\mathrm{dCB}=$ digestibilité in vivo de la cellulose brute ; sur une même ligne, les valeurs marquées d'un même indice sont comparables au seuil de $5 \%$.

celles des blocs, 27,3 et 42,2 g MS $\cdot \mathrm{kg}^{-1} \mathrm{P}^{0.75}$ respectivement par les brebis taries et les agneaux (tableau $V$ ).

\subsubsection{Digestibilité in vivo de la paille}

Les digestibilités in vivo de la paille distribuée seule et complémentée sont présentées dans le tableau IV. Après complémentation, les augmentations sont de 4,5 à 5,4 points pour la dMO, de 8 à 8,9 points pour la dMAT et de 3,1 à 5,8 points pour la dCB.

\subsubsection{Bilan azoté des rations}

L'azote retenu rapporté à l'azote ingéré est pratiquement nul avec la paille distribuée seule $(0,5 \%)$. Il est identique entre la ration $\mathrm{P}+\mathrm{C}(30 \%)$ et la ration $\mathrm{P}+\mathrm{BMN} 2$ $(30 \%)$ et légèrement inférieur avec la ration P+BMN1 (27\%). Les pertes azotées fécales sont plus importantes avec la ration P+BMN1 (45,9\% de l'azote ingéré) alors que les pertes azotées urinaires le sont avec la ration $\mathrm{P}+\mathrm{BMN} 2$ (31,2\% de l'azote ingéré) (tableau VI).

\subsection{Essai 3 : Effet des régimes paille + blocs sur les performances des brebis taries et des agneaux en croissance}

\subsubsection{Variation de poids vif et indice de consommation}

Les brebis recevant les rations $\mathrm{P}+\mathrm{C}$ et $\mathrm{P}+\mathrm{BMN} 1$ ont perdu un peu de poids vif, respectivement $2,2 \mathrm{~g} \cdot \mathrm{j}^{-1}$ et $8,4 \mathrm{~g} \cdot \mathrm{j}^{-1}$ alors que celles recevant la ration $\mathrm{P}+\mathrm{BMN} 2$ en ont gagné un peu, $5,9 \mathrm{~g} \cdot \mathrm{j}^{-1}$ (tableau VII).

Le gain de poids vif le plus élevé est observé chez les agneaux recevant la ration P+BMN2 (105,3 g.j $\left.j^{-1}\right)$ et le plus faible chez ceux recevant la ration $\mathrm{P}+\mathrm{BMN} 1\left(83,6 \mathrm{~g} \cdot \mathrm{j}^{-1}\right)$. L'indice de consommation le plus élevé (14,9 $\mathrm{kg} \mathrm{MS} \cdot \mathrm{kg}^{-1}$ de gain de poids vif) est obtenu chez les agneaux ayant reçu la ration P+BMN1 et le plus faible $\left(10,3 \mathrm{~kg} \mathrm{MS} \cdot \mathrm{kg}^{-1}\right.$ de gain de poids vif) chez les agneaux ayant reçu la ration $\mathrm{P}+\mathrm{C}$ (tableau $V I I)$. 


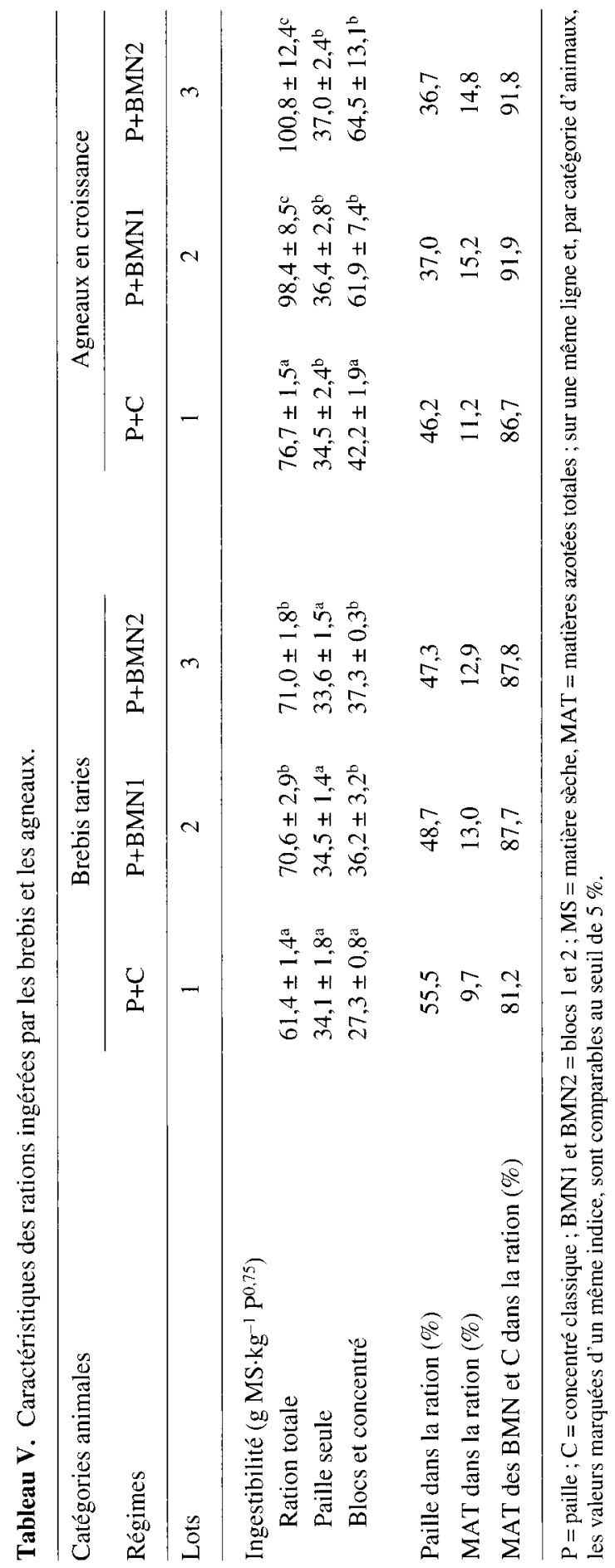


Tableau VI. Bilan azoté des rations (moutons).

\begin{tabular}{lccrr}
\hline g d'azote par jour & $P$ & $\mathrm{P}+\mathrm{C}$ & $\mathrm{P}+\mathrm{BMN1}$ & P+BMN2 \\
\hline Azote ingéré $(\mathrm{Ni})$ & $3,7 \pm 0,2^{\mathrm{c}}$ & $12,6 \pm 0,3^{\mathrm{a}}$ & $12,4 \pm 0,4^{\mathrm{a}}$ & $12,8 \pm 0,1^{\mathrm{a}}$ \\
Azote fécal $(\mathrm{Nf})$ & $3,3 \pm 0,2^{\mathrm{c}}$ & $5,1 \pm 0,7^{\mathrm{a}}$ & $5,7 \pm 0,1^{\mathrm{b}}$ & $5,0 \pm 0,2^{\mathrm{a}}$ \\
Azote urinaire $(\mathrm{Nu})$ & $0,4 \pm 0,1^{\mathrm{d}}$ & $3,7 \pm 0,1^{\mathrm{a}}$ & $3,4 \pm 0,1^{\mathrm{b}}$ & $4,0 \pm 0,1^{\mathrm{c}}$ \\
Azote retenu $(\mathrm{Nr})$ & $0,02 \pm 0,1^{\mathrm{c}}$ & $3,8 \pm 0,1^{\mathrm{a}}$ & $3,3 \pm 0,2^{\mathrm{b}}$ & $3,8 \pm 0,2^{\mathrm{a}}$ \\
$(\mathrm{Nr} / \mathrm{Ni}) \times 100$ & 0,5 & 30 & 27 & 30
\end{tabular}

$\mathrm{P}=$ paille $; \mathrm{BMN} 1$ et $\mathrm{BMN} 2=$ blocs multinutritionnels 1 et $2 ; \mathrm{C}=$ concentré classique $;$ sur une même ligne, les valeurs marquées d'un même indice sont comparables au seuil de $5 \%$.

\subsubsection{Coût alimentaire}

Le coût alimentaire pour l'entretien journalier d'une brebis tarie de $48-49 \mathrm{~kg}$ de poids vif est de 6,6 dinars algériens (DA) avec la ration $\mathrm{P}+\mathrm{C}$ et de 5,5 et 5,7 DA avec les rations $\mathrm{P}+\mathrm{BMN} 2$ et $\mathrm{P}+\mathrm{BMN} 1$. Avec les agneaux en croissance, le coût de l'alimentation par $\mathrm{kg}$ de gain de poids vif est de $66 \mathrm{DA}$ avec la ration $\mathrm{P}+\mathrm{C}$ et de 51 et $58 \mathrm{DA}$ avec les rations $\mathrm{P}+\mathrm{BMN} 2$ et $\mathrm{P}+\mathrm{BMN} 1$. Par rapport aux rations $\mathrm{P}+\mathrm{C}$, les rations renfermant les blocs ont donc permis des économies de 13,6 et $16,7 \%$ sur l'entretien quotidien des brebis taries et de 12,1 et $22,7 \%$ par kg de gain de poids vif avec des agneaux (tableau VII). Cependant, les coûts alimentaires peuvent varier suivant la disponibilité, le prix et les caractéristiques des sousproduits locaux.

\section{DISCUSSION}

Les blocs multinutritionnels utilisés ont une valeur nutritive intéressante ( 21 à $22 \%$ de MAT et 26 à $30 \%$ de MM); ils peuvent ainsi servir de complément pour les pailles et apporter des nutriments pour la flore microbienne du rumen.

Le concentré et les blocs ont augmenté l'ingestion de la paille de $21 \%$. Ce résultat est en accord avec ceux de Kunju [10], Sansoucy et al. [17] et Tiwari et al. [20] pour lesquels la distribution des blocs améliore l'ingestion de la paille de 15 à $30 \%$ et même de $40 \%$ selon Nyarko-Badohu et al. [15].

L'ingestion des blocs multinutritionnels par les brebis taries et les agneaux en croissance est supérieure à celle rapportée chez les petits ruminants par Sansoucy et al. [17], Hadjipanayiotou et al. [7] et Nyarko-badohu et al. [15] : de 3,8 à $16,8 \mathrm{~g} \mathrm{MS} \cdot \mathrm{kg}^{-1} \mathrm{P}^{0.75}$. Cette grande variabilité dans l'ingestion des blocs s'expliquerait par la nature des différentes matières premières incorporées et leur proportion qui influenceraient leur dureté. L'importante consommation des blocs enregistrée dans les essais sur les brebis et les agneaux serait due à leur faible dureté (11\% de ciment contre $15 \%$ dans l'essai de NyarkoBadohu et al. [15]) mais aussi, à la durée de mise des blocs à la disposition des animaux. Cette durée est elle-même liée aux performances animales recherchées.

L'effet améliorateur des blocs (BMN1 et BMN2) sur la dMO de la paille peut être expliqué par l'apport d'éléments catalytiques (matières azotées et minéraux) à la flore microbienne du rumen. Selon Chenost et Kayouli [5], une complémentation de blocs multinutritionnels favorise les fermentations ruminales et, par là, améliore la digestibilité du fourrage ; les blocs utilisés n'ont pas permis une $\mathrm{dCB}$ de la paille supérieure à celle de la paille complémentée avec 


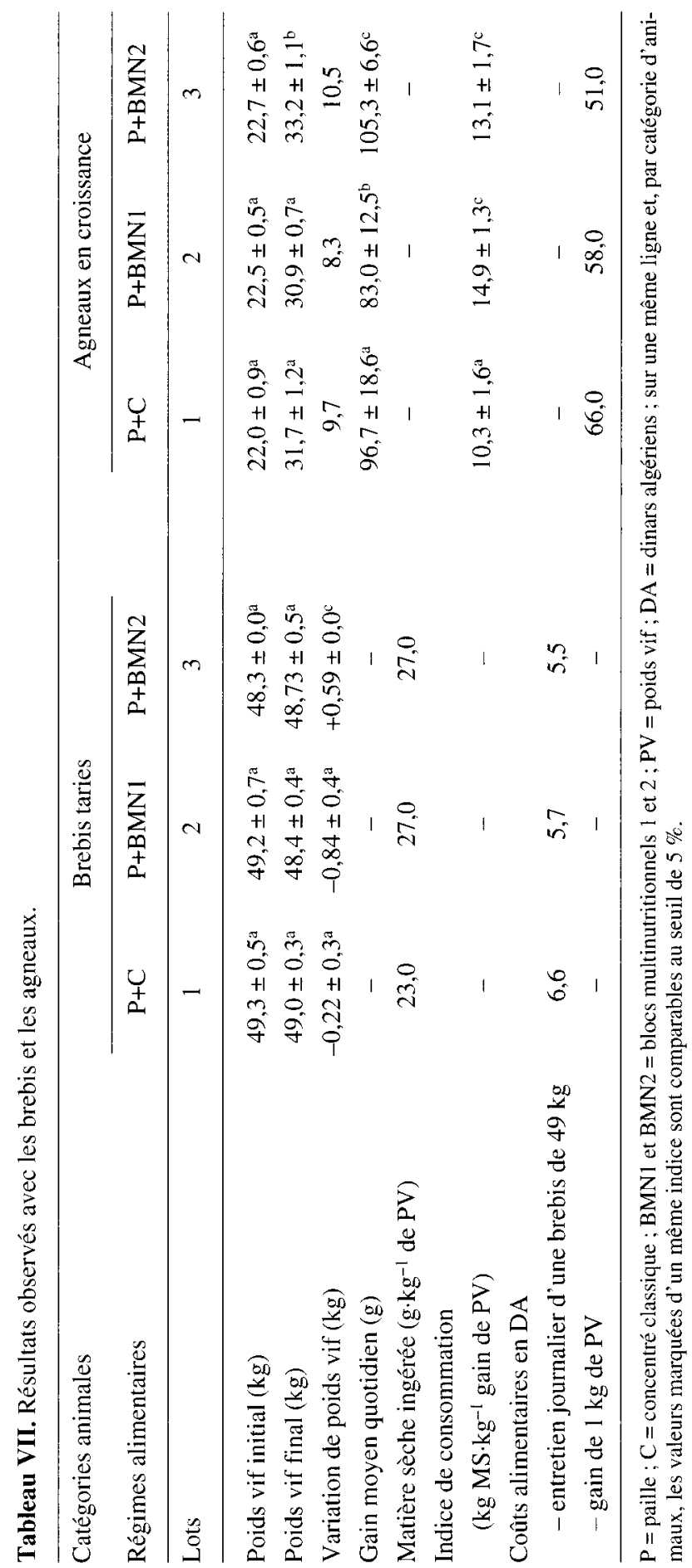


le concentré $(\mathbf{C})$, cela pour deux raisons : les blocs étaient mis à la disposition des animaux seulement 20 min le matin et $20 \mathrm{~min}$ l'après-midi ce qui serait insuffisant pour une ingestion régulière et continue dans la journée pour favoriser une activité cellulolytique optimale par la flore microbienne du rumen $[6,16]$; enfin les blocs BMN1 renferment des grignons riches en parois ligno-cellulosiques peu digestibles.

L'azote des grignons rendu en partie indigestible par les tanins serait à l'origine de la différence de la teneur en azote fécal entre la ration $\mathrm{P}+\mathrm{BMN} 1$ et les rations $\mathrm{P}+\mathrm{C}$ et $\mathrm{P}+\mathrm{BMN}$ 2. Au contraire, la présence des fientes de volaille riches en azote soluble dans les blocs BMN2 (60\% de l'azote des fientes est sous forme soluble selon Nefzaoui [11]) expliquerait l'importante perte azotée urinaire avec la ration $\mathrm{P}+\mathrm{BMN} 2$.

Les faibles performances avec la ration P+BMN1 malgré son ingestion comparable ou supérieure à celle des autres rations, seraient dues à la présence des grignons dans les blocs BMN1 [12] et à la faible digestibilité de sa matière organique et de sa cellulose brute [4], ce qui a limité la digestibilité du bloc BMNl comparé à celle du bloc BMN2. De nombreux auteurs ont rapporté une mauvaise utilisation digestive des grignons d'olives $[2,13,18]$; en raison de la présence de tanins et de lignine.

\section{CONCLUSION}

Les blocs multinutritionnels testés sont intéressants parce qu'ils associent des sousproduits de saveur et d'appétence variables et se caractérisent par une importante valeur nutritive (azote et matières minérales) ; ils peuvent servir de complément pour les pailles.

La complémentation de la paille de blé avec des blocs multinutritionnels renfermant des grignons bruts d'olives ou des fientes de volailles a permis d'améliorer son ingestibilité de $21 \%$ et la digestibilité de sa matière organique de 5,4 points, soit un niveau comparable à celui permis par un concentré classique.

La paille complémentée avec les blocs a permis d'entretenir correctement des brebis taries de 48-49 $\mathrm{kg}$ de poids vif et d'assurer des besoins de croissance modeste à des agneaux. Le bloc renfermant les fientes de volaille est cependant préférable à celui avec les grignons bruts d'olives.

La complémentation avec des blocs multinutritionnels est un moyen pour réduire les coûts alimentaires d'entretien ou de production en évitant, complètement ou partiellement, le recours aux céréales et aux aliments concentrés coûteux, faisant souvent l'objet de spéculation en période de pénurie fourragère.

Les blocs multinutritionnels sont donc appropriés pour utiliser les sous-produits locaux et, sont un moyen efficace pour améliorer la valeur alimentaire de la paille de blé.

\section{RÉFÉRENCES}

11] AOAC, Official Methods of Analysis, 12th ed,, Washington DC, 1975.

[2] Balti M., Incorporation des grignons d'olive dans l'alimentation des ovins et son incidence sur les processus fermentaires au niveau du rumen, mémoire de $3^{c}$ cycle. Inat, Tunisie, 1974 , $105 \mathrm{p}$.

[3] Benahmed H., Dulphy J.P., Note sur la valeur azotée des fourrages pauvres traités à l'urée ou à l'ammoniac, Ann. Zootech. 20 (1985) 335-346.

44 Bencherchali M., Contribution à l'étude de quelques sous-produits agro-industriels algériens : I- caractéristiques chimiques et digestibilité in vitro. II- effets des complémentations à base de sous-produits sur la valeur nutritive de la paille de blé dur, thèse de magister en sciences agronomiques, Inst. Agro. Université de Blida, Algéric, 1994, 118 p.

[5] Chenost M., Kayouli C., Utilisation des fourrages grossiers en régions chaudes, Étude FAO Prod. Santé Anim. 135, 1997, 226 p.

[6] Chesson A., Ørskov E.R., Microbial degradation in the ammoniated feeds in ruminants nutrition, in: Tracer studies on non protein nitrogen for ruminants IAEA-Vienne, FAO, 1984, 153-161. 
17] Hadjipanayiotou M., Labban L., Badran A., Effect of block feeding on the performance of dry Shami cows on poor quality roughages. Document technique no. 2. FAO/UNDP/SYR/003, 1989,26 p.

[8] Hassoun A., Mise au point d'une technique de fabrication de blocs nutritionnels sans mélasse. Rapport de mission effectuée en Tunisie, Division de la production et de la santé animale. FAO/Rome, 1990, 10 p.

[9] Inra, Alimentation des bovins, ovins et caprins. Ouvrage collectif dirigé par R. Jarrige, Ed. Inra, Paris, France, 1988, 476 p.

110| Kunju P.J., Urea molasses block, a feed supplement for ruminants. International workshop on rice straw and related feeds in ruminants rations, March, Sri Lanka, 1986, 12 p.

111] Nefzaoui A., Valeur nutritive des ensilages combinés de fientes de volaille et de grignons d'olives. I- Influence de la durée d'accumulation des fientes et des proportions respectives des fientes et de grignons sur la composition et les caractéristiques fermentaires des ensilages, Ann. Zootech. 40 (1991) 105-111.

[12] Nefzaoui A., Ksaier H., Utilisation de la pulpe d'olive comme aliment de sauvegarde, in : Séminaire International sur la valorisation des sousproduits de l'olivier, Monastir, Tunisie, Dec. 1981.

[13] Nefzaoui A., Hellings P.H., Vanbelle M., Ensiling olive pulp with ammonia: Effects on voluntary intake and digestibility measured by sheep.
34 th. Annual Meeting of the Study Commission EAAP, Madrid, 3-6 October 1983, 7 p.

114| Nefzaoui A., Vanbelle M., Évaluation des méthodes in vitro pour prédire la valeur nutritive des résidus de récolte et des sous-produits agroindustriels des pays en voie de développement, publication $n^{\circ} 37$, laboratoire de biochimie de la nutrition faculté des sciences agronomiques, Louvain-la-Neuve, Belgique, 1984, 38 p.

[15] Nyarko-Badohu D.K., Kayouli C., Ba A.A., Gasmi H., Valorisation des pailles de céréales en alimentation des ovins dans le nord de la Tunisie : traitement à l'urée et à l'ammoniac et complémentation par des blocs mélasse-urée, Options méditerranéennes Série B, Études et Recherches 6 (1994) 129-141.

[16] Preston R.D, Leng R.A., Utilization of tropical feeds by ruminants MTP Press, Lancaster (1984) $621-640$.

[17] Sansoucy R., Aarts G., Preston T.R., Molassesurea blocks as a multinutrient supplement for ruminants. FAO, Animal Production and Health, 72 (1988) 263-278.

[ 18 ] Thériez M., Boule G., Valeur alimentaire du tourteau d'olive, Ann. Zootech. 19 (1970) 143-157.

[19] Tilley J.M.A., Terry R.A., A two-stage technique for the in vitro digestion of forage crops, J. Br. Grasel. Soc. 18 (1963) 104-111.

[20] Tiwari J.P., Singh U.R., Usha Mehra R., Urea molasses mineral blocks as feed supplement: Effect on growth and nutrient utilization in buffalo calves, Ani. Feed Sci. Technol. 29 (1990) 333-341. 